Vol.01/ No. 01

Pages: 21-29

https://www.irojournals.com/iroeea

DOI: https://doi.org/10.36548/jeea.2019.1.003

\title{
HIGH PERFORMANCE NANOWIRE ARRAY BATTERY WITH PRELOADED LITHIUM ION
}

\author{
Nayana Shetty \\ Associate Professor, Department of Electrical and Electronics Engineering, \\ NMAMIT, Nitte, Udupi, Karnataka, India \\ E-mail: nayanar401@gmail.com
}

\begin{abstract}
Electricity playing a major role in the most of the application has become a one of the essential requirement in the existing world. The increased requirement of the electricity has led to the necessity of the rechargeable and the storage batteries and the lithium ion batteries have proved to be an effective in energy storage and the energy conversion for many portable and the implantable devices. Despite the capacities of the lithium ion batteries, the greater pressure on the life time of the battery paves way or the alternative, resulting in the emergence of the nanowires battery arrays. The paper proffers a GaN nanowire arrays integrated with the lithium ion batteries to have high performance Nano wire array -lithium ion battery that inhibits the potentials of the lithium ion battery and the GaN nanowires arrays providing an extended lifetime for the batteries.
\end{abstract}

Keywords: Nano wires, Nano wire battery, lithium ion batteries, GaN nanowires, extended battery life, enhanced performance.

\section{INTRODUCTION}

The continuous progress in the energy requirements has concreted the way for the necessity of the renewable energy sources and the entailment of the storage devices such as batteries [1]. The batteries are a collection of cells with the covering electrical connections with the possible electronics for the control and the protection. The author Thackeray in his research states that the "electrical energy storage systems in the form of batteries are engaged not only as a back-up but also as primary source of power for the for smaller device such as the customer electronics, medical embedding and the power tools used and the toys and he also states that the lithium ion would be an effective alternative to subside the technological issues in the electrical power transportation"[2] the lithium ion batteries, utilizes the similar active material as other batteries for the energy storage and conversion of the energies between the electric power and the chemical storage unit. The table.1 below shows the different types of batteries and their efficiencies along with the energy density and the other attributes. 
Journal of Electrical Engineering and Automation (EEA) (2019)

Vol.01/ No. 01

Pages: 21-29

https://www.irojournals.com/iroeea

DOI: https://doi.org/10.36548/jeea.2019.1.003

\begin{tabular}{|c|c|c|c|c|}
\hline $\begin{array}{l}\text { Energy storage } \\
\text { Technologies }\end{array}$ & Power density & $\begin{array}{l}\text { Energy } \\
\text { density }\end{array}$ & Life Span & Efficiency \\
\hline Fuel Cells & Medium & High & Low/Moderate & Medium \\
\hline Super Capacitors & Very High & Low & High & High \\
\hline Nano Generators & Very low & Unlimited & Unknown & Low \\
\hline Li-ion w/graphite & Medium & Medium & Medium & High \\
\hline Li- ion w/SiNW & Medium & High & Low & High \\
\hline
\end{tabular}

Table .1 Various Energy Storage Technologies Available

The lithium ion being more promising among the devices with the recharge capability, could be further advanced with the improved energy capacity and the increased life cycle by engaging the silicon Nano wires in them. Further the incorporation of the Nano wires in the lithium battery helps it to achieve the better electric conduct and contact with the limited lithium insertion distance. This type of batteries would have prolonged life in the portable devices and the implantable devices. The later researches developed showed that the $\mathrm{SnO}_{2}$ nanowires with the high purity developed utilizing the "self-catalyst growth procedure with the ball-milled mixture" proposed by park et al enabled to overcome the deterioration caused by the conventional catalyst and to furthermore increase the production [4]. Many research have developed batteries with the incorporation of nanowires such as the manganese oxide electrodes made of interconnected nanowires to show a larger energy storage capacity compared to the traditional graphite constituents for the lithium batteries [5], integration of the Si-Cu nanowires with lithium ion battery to have an improved cycling stability and an higher capability as a superior anode.

The paper also proposes a high performance nanowire array batteries preloaded with the lithium ion to enhance the storage capability of the battery by enhancing the discharge time and prolonging the battery life.

The remaining of the paper is arranged with the related works detailing the lithium ion batteries and the necessary updation in it using the Nano wires in the section 2 and the proposed work explaining the capabilities of the Nano wires and the efficiency of the Nano wire batteries along with the performance enhancement with the nanowire array batteries preloaded with the lithium ion in the section 3. The result evaluation is presented in the section 4 and the conclusion in the section 5 . 
Journal of Electrical Engineering and Automation (EEA) (2019)

Vol.01/ No. 01

Pages: 21-29

https://www.irojournals.com/iroeea

DOI: https://doi.org/10.36548/jeea.2019.1.003

\section{RELATED WORKS}

The storage devices being essential equipment in the world nowadays, the author Brandt et al [1] explains areas were the lithium ion batteries could be applied with. Thackeray et al [2] the paper details the lithium ion batteries in the storage to be applied for the transportations necessities. Chan et al [3] the paper details the lithium ion batteries upgraded to an improved energy capacity and the increased life cycle by employing the silicon nanowires in them. Park et al [4] the paper details the "self-catalyst growth procedure with the ball-milled mixture" for the achieving an appropriate $\mathrm{SnO}_{2}$ Nano wire with the high purity. Wu, et al [5] explores the suitability of the synthesis of the manganese oxide electrodes with the interconnected nanowire structures as anode for the lithium ion batteries that are rechargeable. Shen et al [6] the paper clearly detailing the improved conductivity in the batteries by utilizing the $\mathrm{Li}_{4} \mathrm{Ti}_{5} \mathrm{O}_{12}$ Nano wire arrays to enhance the speed of the chemical reaction, enabling a outstanding performance, and an extended life. Cao et al [7] the $\mathrm{Cu}-\mathrm{Si} \mathrm{Nano}$ wire with high rate anode materials are utilized with the lithium-ion batteries to enhance the cycling stability and the capability. Reddy et al [8] elaborates the principles of the battery operation along with the details of the primary, the secondary batteries, the specialized batteries, fuel cells and the electrochemical capacitors. Lankey et al [9] the paper compares the performance of the rechargeable batteries using the life cycle methods. $\mathrm{Zu}$, et al [10] provides details of the thermodynamic analysis of the densities of the batteries in relation to the energy. Calarco et al [11] elaborates the growth and the nucleation of the GaN nanowires on $\mathrm{Si}$ utilizing the molecular beam epitaxy. Lepsa et al [12] show the growth and the characterization of the "Core shell nanowires".

\section{PROPOSED WORK}

\subsection{LITHIUM ION BATTERY}

The existing industry and the other activities related with the energy depletion are dominated by the lithium ion batteries as they prevail with the absolute capability of holding huge volume of energy per weight. Lithium-ion battery is a sort of rechargeable battery that is prominent among the portable devices and the electric-vehicle, further gaining an increasing popularity in army and the aerospace utilizations. The charging and the discharging in the 
Journal of Electrical Engineering and Automation (EEA) (2019)

Vol.01/ No. 01

Pages: 21-29

https://www.irojournals.com/iroeea

DOI: https://doi.org/10.36548/jeea.2019.1.003

lithium ion batteries totally depend on the movement of the lithium ions to and fro between the positive electrode and the negative electrodes respectively. The lithium ion batteries possess a higher energy density, with the low self-discharge and are also explosive if erroneously charged.

The lithium batteries are constructed with the three principal components that are negative electrode, positive electrode and the electrolyte. The components of the battery are usually made of different materials for different batteries as the choices of the materials play a vital role in the voltage, battery life, energy density and the safety.

These lithium batteries are very costly but the function even in at broader range of the temperatures along with the energy densities that are very much higher. The figure .1 below shows the battery pack of the lithium ion batteries along with the protecting circuit to limit the peak voltage, observe the status of charging and the flow of the current, record the charge capacity, and observing the temperature.

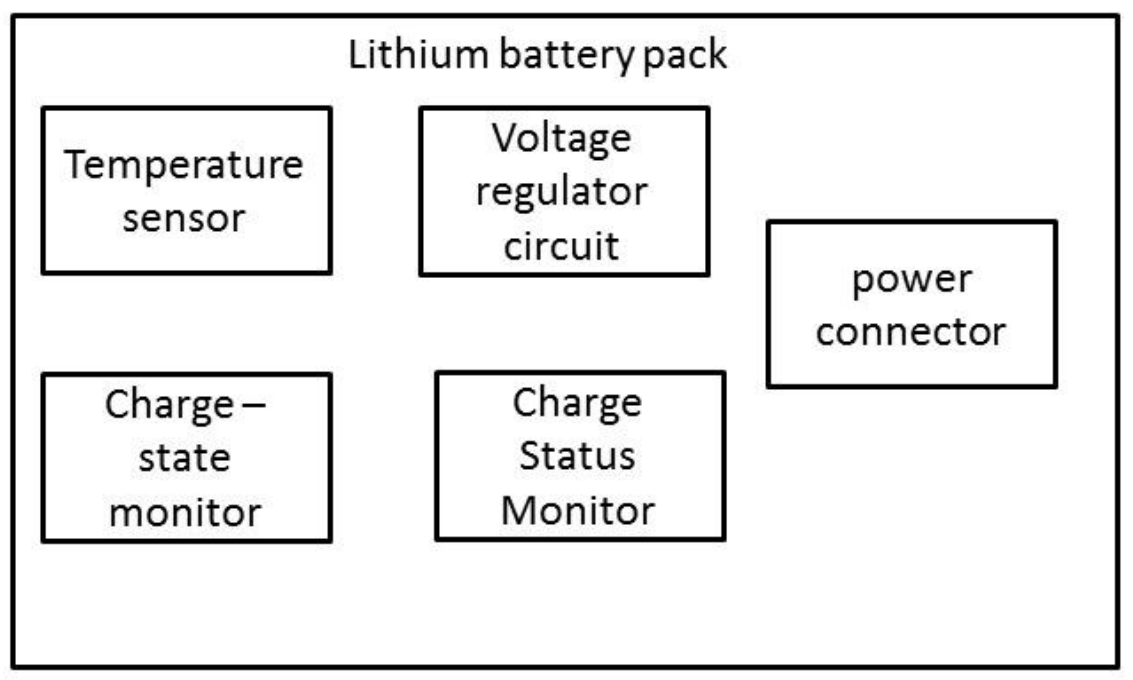

Fig .1 The Battery Pack of Lithium -ion Battery

Despite its abilities of holding enormous amount of energy to satisfy the user requirements, they suffer from the complications in the charging requirements and the reduced life time. This makes the lithium-ion battery incompatible for the devices that are implantable. The entailment in the enhancement of the battery lifetime has even led to the use of the Nanotechnology in them. 
Journal of Electrical Engineering and Automation (EEA) (2019)

Vol.01/ No. 01

Pages: 21-29

https://www.irojournals.com/iroeea

DOI: https://doi.org/10.36548/jeea.2019.1.003

\subsection{NANO WIRE BATTERY}

The proposed work aims in enhancing the storage capabilities and improve the conductivity and the discharging time to prolong the life of the batteries using the high performance Nanowire battery arrays with the lithium ion. Starting from the basic the Nano wires in the size that is in between the microscopic and the molecular ranges in the diameter of order of a nanometer $\left(10^{-9}\right.$ meters) enable a simple and an efficient battery design and manufacturing at ease. They are wires of smaller sizes that knock into the properties of the quantum mechanics. The Nano wire batteries utilize the Nano wires in order to increase the surface area of its electrodes. As the Nano wire are of very small size they ensure the more surface area to be achieved in the smaller volume in return allowing a huge amount of charge to be kept at reserve. Though the Nanowires do possess certain capabilities by increasing the storage capacity they suffer from the cycle instability or breakdown on repeated charging. To improve the Nano wires stability from the continuous break downs caused by the repeated charging.

The lithium batteries though seeming to be ubiquitous among the portable devices and mobile technologies faces, limitations in the battery life time. So this makes the mobile electronics to go with the Nano wire batteries that could provide with the better lifespan compared to the lithium ion batteries. The proposed method aims in developing the batteries combining the Nanowire and the lithium ion batteries overcoming the problem of the lifespan in the lithium ion batteries and the eluding the problem of instability in the nanowires by introducing the GaN Si nanowires that provide with the performance improvements in the batteries in terms of the stability and the life time.

\subsection{PROPOSED METHOD OF GAN Si NANO WIRE -LITHIUM ION BATTERIES}

The Proposed method utilizes the GaN Si Nano wire by growing the GaN Nano wires on the Si utilizing the molecular beam epitaxy [11] to develop high performance Nano wire- lithium ion battery. The fig. 2 below show the proposed method of the GaN Si Nano wire arrays in the lithium batteries to overcome the problem of the life time in the batteries and the instability. 
Vol.01/ No. 01

Pages: 21-29

https://www.irojournals.com/iroeea

DOI: https://doi.org/10.36548/jeea.2019.1.003
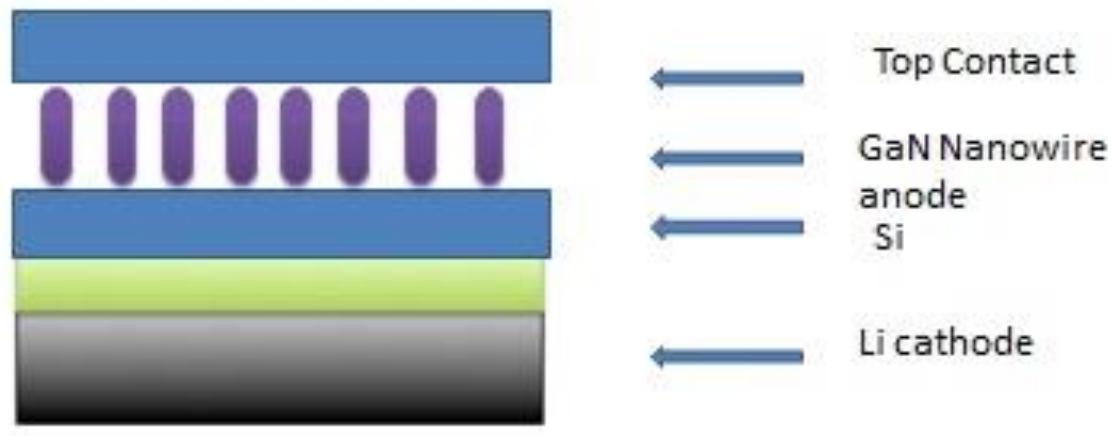

Fig .2 Proposed High performance Battery.

The battery framed utilizing the GaN Nanowires grown over the $\mathrm{Si}$ is used as the anode in the lithium batteries allows a better deposition of charges, with the more space allotted for the charging storage due to the usage of the nano wires, the GaN Si deposited over the Si allows to have a lower discharge rate and improvisation in the life time of the batteries along with the stability that is necessitated in the nano wires. This GaN nanowire growth process utilizing the molecular beam epitaxy [11] over the $\mathrm{Si}$, decreases the nanowires densities to $3 * 10^{9} \mathrm{NWs} / \mathrm{cm}^{2}$. Thus GaN nanowire grown over the Si shows improvement in maximizing the storage space for the energy making the batteries durable for an even longer time compared to the conventional lithium-ion batteries. This GaN Nano Wire $\mathrm{Si}$ arrays for the Li-ion batteries proves to be an effective alternative for the conventional $\mathrm{Li}$-ion batteries. The proposed methodology provides with a better enhancement in the life time of the battery with the evading of the instability in the batteries to the continuous charging and the discharging.

\section{RESULTS}

The GaN Si Nano wire battery is applied to the various portable devices to know the discharge time enhancements and the lifetime enhancements achieved. These batteries are engaged in portable devices, mobile devices and the implantable medical devices to know about the discharge status, the charging time of the GaN Si Nano wire-Li-ion batteries and their prolonged lifetime. Further the results obtained are compared with the prevailing lithium-ion battery to high light the proficiency of the GaN Si Nano wire-Li-ion batteries. 
Journal of Electrical Engineering and Automation (EEA) (2019)

Vol.01/ No. 01

Pages: 21-29

https://www.irojournals.com/iroeea

DOI: https://doi.org/10.36548/jeea.2019.1.003

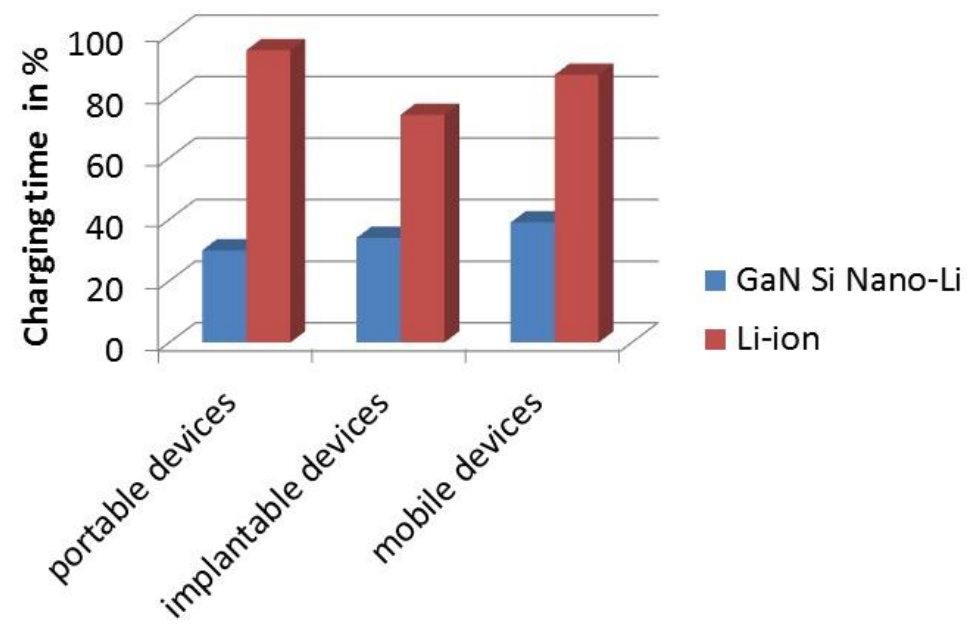

Fig. 3 charging Time required

The fig. 3 above shows the percentage of the charging time required for the GaN Si Nano wire-Li-ion battery and the lithium-ion battery for the various portable devices, mobile devices and the implantable devices. The results obtained shows that the performance of the GaN Si Nano wire-Li-ion battery is very much high with the speedy charging capabilities compared to the lithium-ion battery.

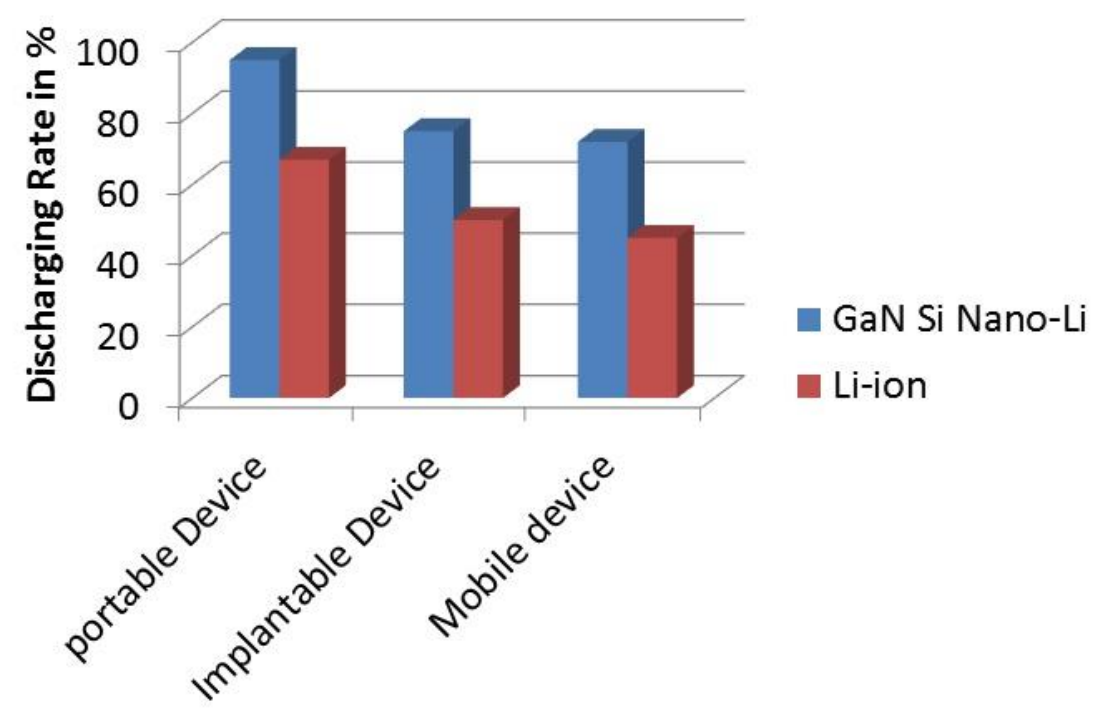


Journal of Electrical Engineering and Automation (EEA) (2019)

Vol.01/ No. 01

Pages: 21-29

https://www.irojournals.com/iroeea

DOI: https://doi.org/10.36548/jeea.2019.1.003

Fig .4 Discharging Time

The fig. 4above shows that the discharging rate of the GaN Si Nano wire-Li-ion battery and the lithium-ion battery for the various portable devices, mobile devices and the implantable devices. The results attained shows that the performance of the GaN Si Nano wire-Li-ion battery is very much high with the very discharging rate is compared to the lithium-ion battery.

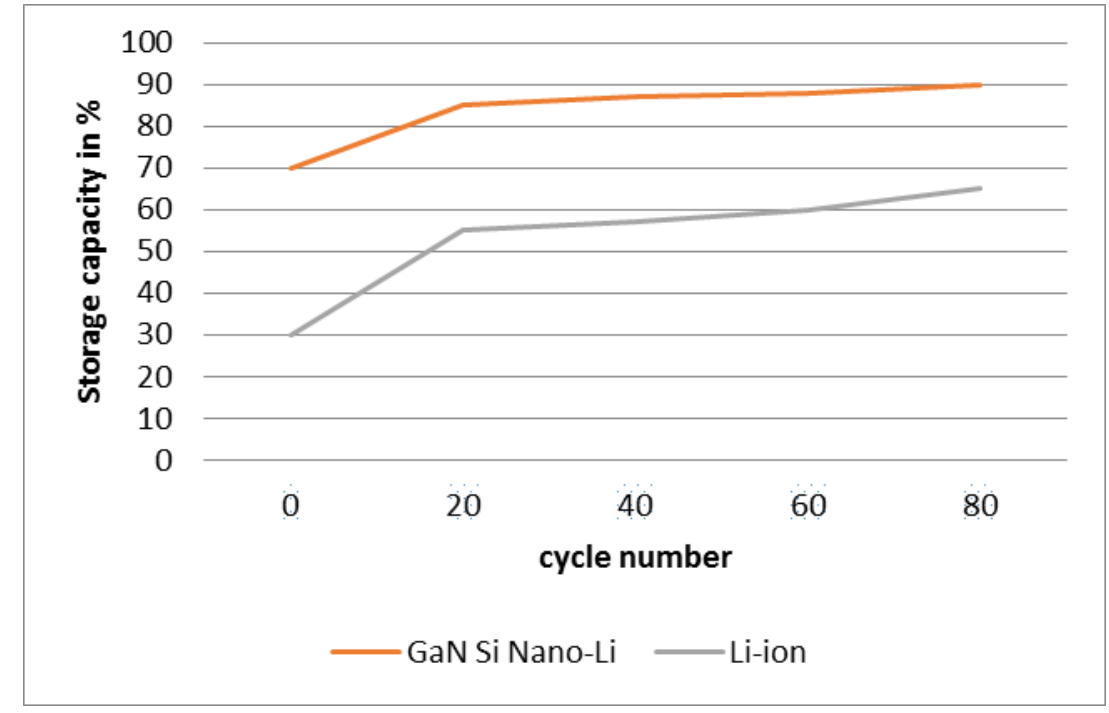

Fig .5 Storage Capacity

The fig. 5 above shows the storage capacity of the batteries GaN Si Nano wire-Li-ion and the lithium-ion battery. The storage capacity of the proposed is very much increased compared to the lithium-ion battery. The result acquired shows a 35\% higher storage capacity of 35\% higher compared to the lithium-ion battery.

\section{CONCLUSION}

The electricity being a non -renewable resources and a very essential requirement in the present days, requires an effective accumulators that could have a higher storage capacity, a lower discharge time and a lengthier life. So the paper proposes the a battery with the higher storage capacity and lengthier life and a lower discharge rate by embedding the GaN Si Nano wires into the lithium -ion batteries. The results acquired using the GaN Si Nano wire- 
Journal of Electrical Engineering and Automation (EEA) (2019)

Vol.01/ No. 01

Pages: 21-29

https://www.irojournals.com/iroeea

DOI: https://doi.org/10.36548/jeea.2019.1.003

Li-ion and the ordinary lithium-ion battery by engaging them with the portable, implantable and the mobile devices

show that the GaN Si Nano wire-Li-ion batteries show an improved performance than the conventional lithium -ion batteries.

\section{References}

[1] Brandt, Klaus. "Fields of application for lithium-ion batteries." In Lithium-Ion Batteries: Basics and Applications, pp. 359-368. Springer, Berlin, Heidelberg, 2018.

[2] Thackeray, Michael M., Christopher Wolverton, and Eric D. Isaacs. "Electrical energy storage for transportation-approaching the limits of, and going beyond, lithium-ion batteries." Energy \& Environmental Science 5, no. 7 (2012): 7854-7863.

[3] Chan, Candace K., Hailin Peng, Gao Liu, Kevin Mcllwrath, Xiao Feng Zhang, Robert A. Huggins, and Yi Cui. "High-performance lithium battery anodes using silicon nanowires." Nature nanotechnology 3, no. 1 (2008): 31.

[4] Park, Min-Sik, Guo-Xiu Wang, Yong-Mook Kang, David Wexler, Shi-Xue Dou, and Hua-Kun Liu. "Preparation and electrochemical properties of $\mathrm{SnO} 2$ nanowires for application in lithium-ion batteries." Angewandte Chemie International Edition 46, no. 5 (2007): 750-753.

[5] Wu, Mao-Sung, Pin-Chi Julia Chiang, Jyh-Tsung Lee, and Jung-Cheng Lin. "Synthesis of manganese oxide electrodes with interconnected nanowire structure as an anode material for rechargeable lithium ion batteries." The Journal of Physical Chemistry B 109, no. 49 (2005): 23279-23284.

[6] Shen, Laifa, Evan Uchaker, Xiaogang Zhang, and Guozhong Cao. "Hydrogenated Li4Ti5O12 nanowire arrays for high rate lithium ion batteries." Advanced Materials 24, no. 48 (2012): 6502-6506.

[7] Cao, Fei-Fei, Jun-Wen Deng, Sen Xin, Heng-Xing Ji, Oliver G. Schmidt, Li-Jun Wan, and Yu-Guo Guo. "Cu-Si Nanocable Arrays as High-Rate Anode Materials for Lithium-Ion Batteries." Advanced materials 23, no. 38 (2011): 4415-4420.

[8] Reddy, Thomas B. Linden's handbook of batteries. Vol. 4. New York: McGraw-hill, 2011.

[9] Lankey, Rebecca L., and Francis C. McMichael. "Life-cycle methods for comparing primary and rechargeable batteries." Environmental science \& technology 34, no. 11 (2000): 2299-2304.

[10]Zu, Chen-Xi, and Hong Li. "Thermodynamic analysis on energy densities of batteries." Energy \& Environmental Science 4, no. 8 (2011): 2614-2624.

[11]Calarco, Raffaella, Ralph J. Meijers, Ratan K. Debnath, Toma Stoica, Eli Sutter, and Hans Lüth. "Nucleation and growth of GaN nanowires on $\mathrm{Si}$ (111) performed by molecular beam epitaxy." Nano letters 7, no. 8 (2007): 2248-2251. 
Journal of Electrical Engineering and Automation (EEA) (2019)

Vol.01/ No. 01

Pages: 21-29

https://www.irojournals.com/iroeea

DOI: https://doi.org/10.36548/jeea.2019.1.003

[12] Lepsa, Mihail Ion, Gunjan Nagda, Pujitha Perla, Nataliya Demarina, and Detlev Grützmacher. "InAs/GaSb

Core-Shell Nanowires: Growth and Characterization." In 2019 Compound Semiconductor Week (CSW), pp.

1-1. IEEE, 2019. 